OPEN ACCESS

Edited by:

Hui-Jia Li,

Beijing University of Posts and

Telecommunications (BUPT), China

Reviewed by:

Lin Wang,

University of Cambridge,

United Kingdom

Chengyi Xia,

Tianjin University of Technology, China

${ }^{*}$ Correspondence:

Zhoujun LI

lizi@buaa.edu.cn

Specialty section:

This article was submitted to

Social Physics,

a section of the journal

Frontiers in Physics

Received: 25 January 2021

Accepted: 08 March 2021

Published: 11 May 2021

Citation:

Liu H, Wang X, Liu L and Li Z (2021)

Co-evolutionary Game Dynamics of

Competitive Cognitions and Public

Opinion Environment

Front. Phys. 9:658130.

doi: 10.3389/fphy.2021.658130

\section{Co-evolutionary Game Dynamics of Competitive Cognitions and Public Opinion Environment}

\author{
Haoyan Liu ${ }^{1}$, Xin Wang ${ }^{1,2,3}$, Longzhao Liu ${ }^{1,2,3,4}$ and Zhoujun Li ${ }^{1,5 *}$ \\ ${ }^{1}$ State Key Lab of Software Development Environment, Beihang University, Beijing, China, ${ }^{2}$ Key Laboratory of Mathematics, \\ Informatics and Behavioral Semantics (LMIB), Advanced Innovation Center of Big Data and Brain Computing (BDBC), School \\ of Mathematical Sciences, Beihang University, Beijing, China, ${ }^{3}$ PengCheng Laboratory, Shenzhen, China, ${ }^{4}$ ShenYuan Honor \\ School, Beihang University, Beijing, China, ${ }^{5}$ College of Computer Science and Software Engineering, Shenzhen University, \\ Shenzhen, China
}

Competitive cognition dynamics are widespread in modern society, especially with the rise of information-technology ecosystem. While previous works mainly focus on internal interactions among individuals, the impacts of the external public opinion environment remain unknown. Here, we propose a heuristic model based on co-evolutionary game theory to study the feedback-evolving dynamics of competitive cognitions and the environment. First, we show co-evolutionary trajectories of strategy-environment system under all possible circumstances. Of particular interest, we unveil the detailed dynamical patterns under the existence of an interior saddle point. In this situation, two stable states coexist in the system and both cognitions have a chance to win. We highlight the emergence of bifurcation phenomena, indicating that the final evolutionary outcome is sensitive to initial conditions. Further, the attraction basins of two stable states are not only influenced by the position of the interior saddle point but also affected by the relative speed of environmental feedbacks.

Keywords: co-evolutionary game theory, feedback-evolving dynamics, competitive cognitions, public opinion environment, strategy-environment system

\section{INTRODUCTION}

Individual cognitive process and public cognition evolution have aroused great concern and have been widely studied in recent years due to the rapid development of information-technology ecosystem consisting of news medias, blogs, large-scale social networks, etc. [1-6]. Throughout this paper, "cognition" is a psychological representation that can be any piece of "knowledge" an individual holds, including information, attitude, beliefs, and behaviors, in accordance with Festinger's definition $[7,8]$. In modern society, online social networks reshape the way of public discourse and cognition consumption patterns, amplify sociocognitive biases, which further influences the evolution of public opinions or collective behaviors [9-13]. A recent work reveals that the combination of external political campaigns (or mass medias) and online social influence processes could give rise to partisan echo chambers [14]. Complicating matters further, the complex interplay between different social networks could also affect cognition diffusion $[15,16]$, leading to the emergence of rich and important physical phenomena such as non-monotonic promotion [17], phase transition [18], and bistability [19], which has been demonstrated by numerous studies using multiplex networks [20-23]. 
Of particular interest, an important line of research focuses on competitive cognition dynamics, which mimic the ubiquitous scenarios where individuals are confronted with multiple and mutually exclusive cognitions [24-26]. Understanding the spreading processes as well as predicting the evolutionary outcomes of competitive cognitions is of fundamental significance for overcoming a series of realworld challenges, ranging from public health problems, social polarization to economic development [27-29]. For instance, pro-vaccination vs. anti-vaccination beliefs, adopting social distancing behaviors during COVID-19 pandemic or not, Biden vs. Trump, supporting the use of new technologies or not, truths vs. rumors, etc. [30-32]. Originally, the competing dynamics were considered in the field of infectious disease, where the classic susceptible-infected-susceptible (SIS) model and susceptible-infected-recovered (SIR) model are extended to study the co-contagion process of two competitive diseases [3335]. These epidemic-like models were subsequently extended to describe the competitive information diffusion [36, 37]. Further, the complex contagion models that incorporate the complicated behavioral patterns were also developed, along with the emerging empirical studies exploring dynamical characteristics via real data [38-40].

While previous models interpret the evolution of competitive cognitions mainly as a result of internal interactions among individuals, either described by the effects of social influence or by the spreading dynamics such as contact-based transmission, the impact of external public opinion environment, especially the media atmosphere of supporting a certain cognition, is ignored [14]. For example, when exposed to a new cognition, the increasing number of adopters or supporters may attract the attention of medias and promotes the supportive level of public opinion environment. In turn, an ascending public opinion environment could encourage more people to adopt or support a new cognition, providing positive evidence for the supporters in public discourse. Therefore, the evolutionary outcome of the competitive cognitions is actually determined by both the benefit from the new cognition and the feedback from public opinion environment, especially in the era of social medias. However, it remains largely unknown how the public opinion environment influences the evolutionary dynamics of competitive cognitions.

To advance this important issue, in this paper, we propose a theoretical framework to describe the co-evolution of competitive cognitions and public opinion environment using co-evolutionary game theory, which has been developed recently to study the feedback loops between environment and strategies that are widely observed in biological and ecological systems [4146]. The "cooperators" are defined as individuals who support the new cognition while the "defectors" are those who support the old cognition. Hence, the evolution of competitive cognitions can be characterized by the replicator dynamics in evolutionary game theory. The core idea of our model is to incorporate the impact of environmental feedback into cognition dynamics through an additional payoff from public discourse. Conversely, the external public opinion environment is a function of the current proportions of cooperators and defectors. First, we conduct theoretical analysis for the stability of all fixed points and present co-evolutionary dynamics of cooperation and environment under different situations. We find that cooperation or defection can be the sole dominant strategy among population, i.e., one of the cognitions always wins the competition, but only if the benefit or the loss from adopting the new cognition exceeds a threshold related to the influence of public discourse. In other cases, the system has a saddle point and the two cognitions both have a chance to survive, depending on the initial conditions. We further explore the dynamical properties under the existence of an interior saddle point and find the emergence of bifurcation phenomena. In other words, the evolutionary directions of the co-evolution system can be changed through a tiny disturbance, which indicates the existence of "randomness" or "luckiness" in real-world cognition competitions, making it difficult to steer the evolution to a desired direction $[46,47]$. Furthermore, we show how the parameters in our model affect the position of the interior saddle point and that the relative feedback speed of public opinion not only influences the convergence speed but also changes the attraction basin of the two stable states. Finally, we incorporate systematic stochasticity into our deterministic model and reveal the detailed impact of stochastic environmental fluctuations.

\section{MODEL}

In this section, we introduce a modeling framework of twostrategy co-evolutionary game in a well-mixed population to characterize the competition of two cognitions under the influence of modern information-technology ecosystem. When exposed to two cognitions, an individual can choose either to support the new cognition (be a cooperator) or support the old cognition (be a defector). The cooperator receives a fixed payoff $P_{c}$ from the new cognition and takes the risk of "innovation" $c$, while the defector receives $P_{d}$ for adopting the old cognition. Meanwhile, the player receives an extra payoff or loss during public discourse, the value of which is decided by the supportive level for the two cognitions from external environment, i.e., public opinion environment $n, n \in[0,1]$. The schematic of our model is illustrated in Figure 1. The payoff matrix of this game can be written as

$$
A=\left[\begin{array}{cc}
P_{c}-c+n k_{1} & P_{c}-c-k_{2}((1-n)-n) \\
P_{d}-k_{2}(n-(1-n)) & P_{d}+(1-n) k_{1}
\end{array}\right]
$$

where $n$ and $1-n$ denote the environments for supporting the new cognition and the old cognition, and $k_{1}, k_{2}>0$ are the rates of receiving payoffs/losses from homogeneous interactions and heterogeneous conflicts during public discourse, respectively. For example, if a cooperator plays with a cooperator, they talk to each other and both satisfy with the discussion, receiving $n k_{1}$ from the interaction in addition to $P_{c}-c$ from the new cognition itself. If a cooperator plays with a defector, however, they debate each other and the virtual conflict makes the cooperator loses $k_{2}((1-n)-n)$. Note that here $((1-n)-n)$ indicates the current environmental advantage of defectors over cooperators. If $((1-n)-n)<0$, the cooperator actually wins the argument 


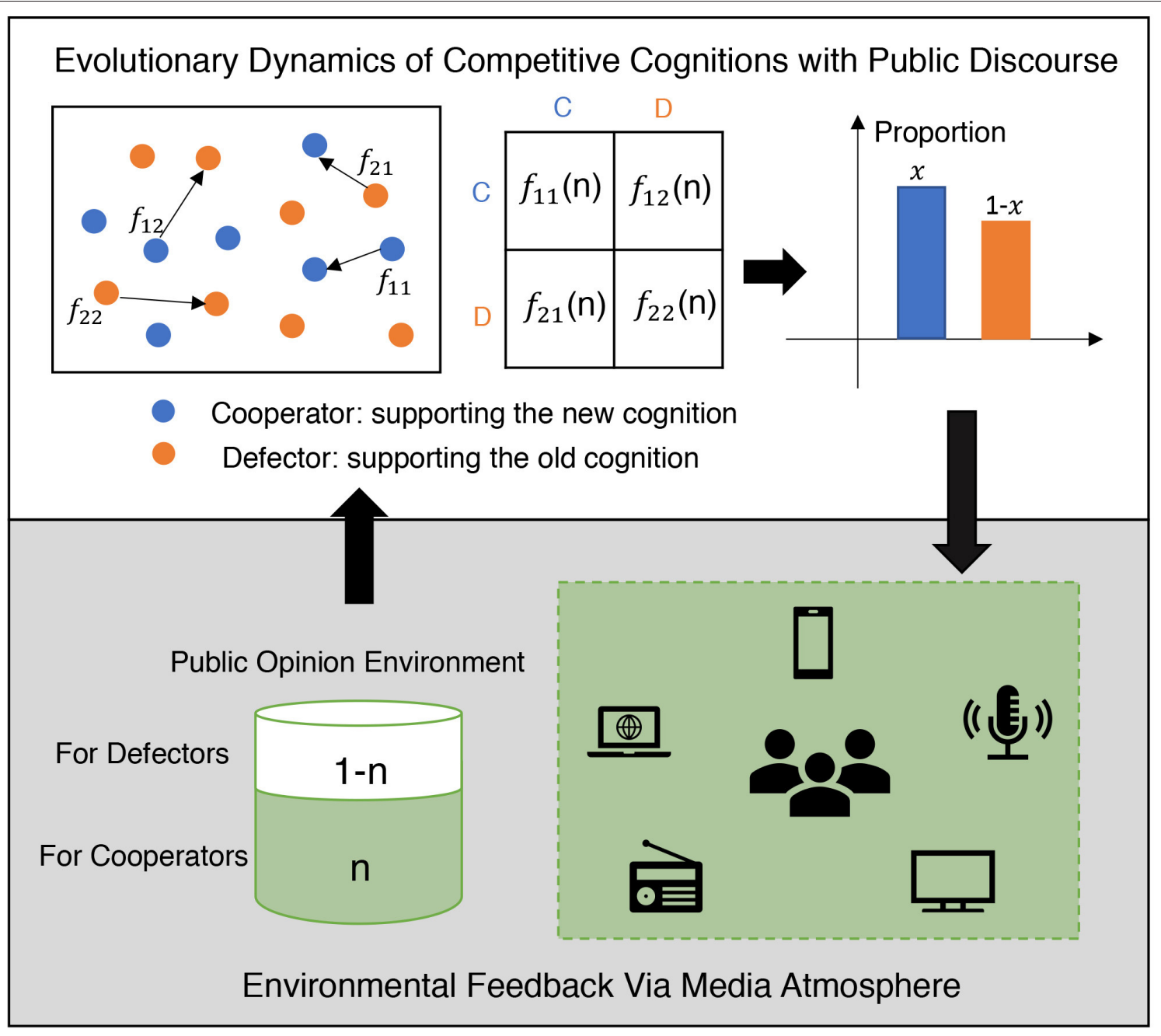

FIGURE 1 | Schematic of our co-evolutionary modeling framework. (Top) The evolution of competitive cognitions, described by the fraction of cooperators who support the new cognition, is determined by a payoff matrix. (Top, Bottom) The fraction of cooperators $x$ modifies the public opinion environment $n$, which in turn influences the payoffs $f_{i j}$.

and receives a positive benefit. Thus, the fitnesses of cooperators and defectors, $f_{c}$ and $f_{d}$, are

$$
\left\{\begin{array}{l}
f_{c}=\left(P_{c}-c+n k_{1}\right) x+\left(P_{c}-c-k_{2}(1-2 n)\right)(1-x) \\
f_{d}=\left(P_{d}-k_{2}(2 n-1)\right) x+\left(P_{d}+(1-n) k_{1}\right)(1-x)
\end{array}\right.
$$

The standard replicator dynamics for the fraction of cooperators $x$ is

$$
\begin{aligned}
\dot{x} & =x\left(f_{c}-\bar{f}\right) \\
& =x(1-x)\left(f_{c}-f_{d}\right) \\
& =x(1-x)\left(k_{1} x+\left(k_{1}+2 k_{2}\right) n+P_{1}-k_{1}-k_{2}\right)
\end{aligned}
$$

where $\bar{f}=x f_{c}+(1-x) f_{d}$ represents the average fitness of the population, $P_{1}=P_{c}-c-P_{d}$ indicates the inherent fixed payoff of cooperation (adopting the new cognition) vs. defection (adopting the old cognition). Meanwhile, the public opinion environment is modified by the population states, and the environmental evolution is given by

$$
\dot{n}=\epsilon n(1-n)(\theta x-(1-x))
$$

where $\epsilon$ denotes the relative feedback speed of the environment compared to cognition dynamics. The logistic term $n(1-n)$ ensures $n \in[0,1]$. And $\theta>0$ is the relative strength of enhancing the environment by cooperators vs. decreasing the environment by defectors.

Therefore, the co-evolutionary game dynamics of competitive cognitions and public opinion environment can be written as follows:

$$
\left\{\begin{array}{l}
\dot{x}=x(1-x)\left(k_{1} x+\left(k_{1}+2 k_{2}\right) n+P_{1}-k_{1}-k_{2}\right) \\
\dot{n}=\epsilon n(1-n)(\theta x-(1-x))
\end{array}\right.
$$




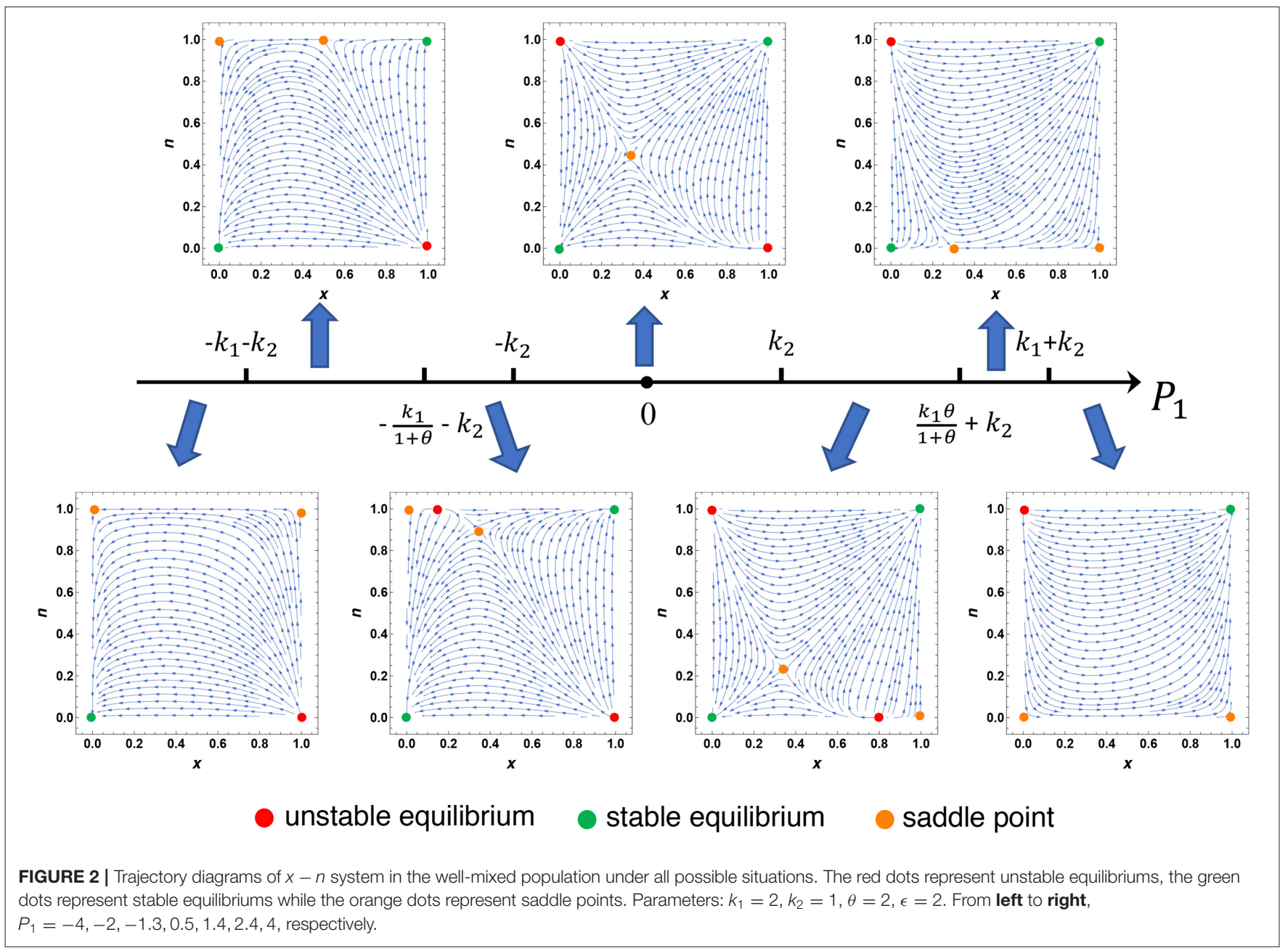

\section{RESULTS}

\subsection{Co-evolution of Cooperation and Environment Under Different Circumstances}

First, we provide trajectory diagrams of $x-n$ system to illustrate co-evolutions of cooperation and environment in the wellmixed population under all possible circumstances in Figure 2. Since the environment $n$ is confined in $[0,1]$, all parameters should naturally be in the same order of magnitude. In order to provide a typical case close to reality, we assume the rate of receiving payoffs from homogeneous interactions $\left(k_{1}\right)$ is larger than that from heterogeneous conflicts during public discourse $\left(k_{2}\right)$. And cooperators have advantages over defectors in terms of enhancing the environment (i.e., $\theta \geq 1$ ), which means the new cognition or social innovation is favored by the media atmosphere. In addition, we assume the environment evolves faster than cognition dynamics $(\epsilon \geq 1)$. For simplicity and without loss of generality, we set $k_{1}=2, k_{2}=1, \theta=2$, $\epsilon=2$ and change $P_{1}=-4,-2,-1.3,0.5,1.4,2.4,4$, respectively. There are seven possible fixed points in the system: four locate in the corners, two on the boundary and the remaining one is an interior fixed point. The detailed theoretical analysis for the existence conditions and the stability of these fixed points are provided in Supplementary Materials.

When $P_{1}>k_{1}+k_{2}$, the system has four corner fixed points, within which $(1,1)$ is the only stable equilibrium, corresponding to the state where the population is dominated by cooperators and the external environment fully supports the new cognition. Similarly, when $P_{1}<-k_{1}-k_{2},(0,0)$ is the only stable equilibrium and the old cognition will win the competition with the environment reaches its minimum value.

When $-k_{1}-k_{2}<P_{1}<k_{1}+k_{2}$, however, $(0,0)$ and $(1,1)$ are both stable equilibriums and the final state of the system depends on initial conditions. More specifically, when $-k_{1}-k_{2}<$ $P_{1}<-\frac{k_{1}}{1+\theta}-k_{2}$ or $\frac{\theta k_{1}}{1+\theta}+k_{2}<P_{1}<k_{1}+k_{2}$, there exists a boundary saddle point $\left(\frac{-k_{2}-P_{1}}{k_{1}}, 1\right)$ or $\left(\frac{k_{1}+k_{2}-P_{1}}{k_{1}}, 0\right)$ in addition to four corner fixed points, respectively. And the phase plane is divided into two regions: one evolves to $(0,0)$ and one evolves to $(1,1)$.

In particular, when $-\frac{k_{1}}{1+\theta}-k_{2}<P_{1}<\frac{\theta k_{1}}{1+\theta}+k_{2}$, we find the emergence of an interior saddle point: 

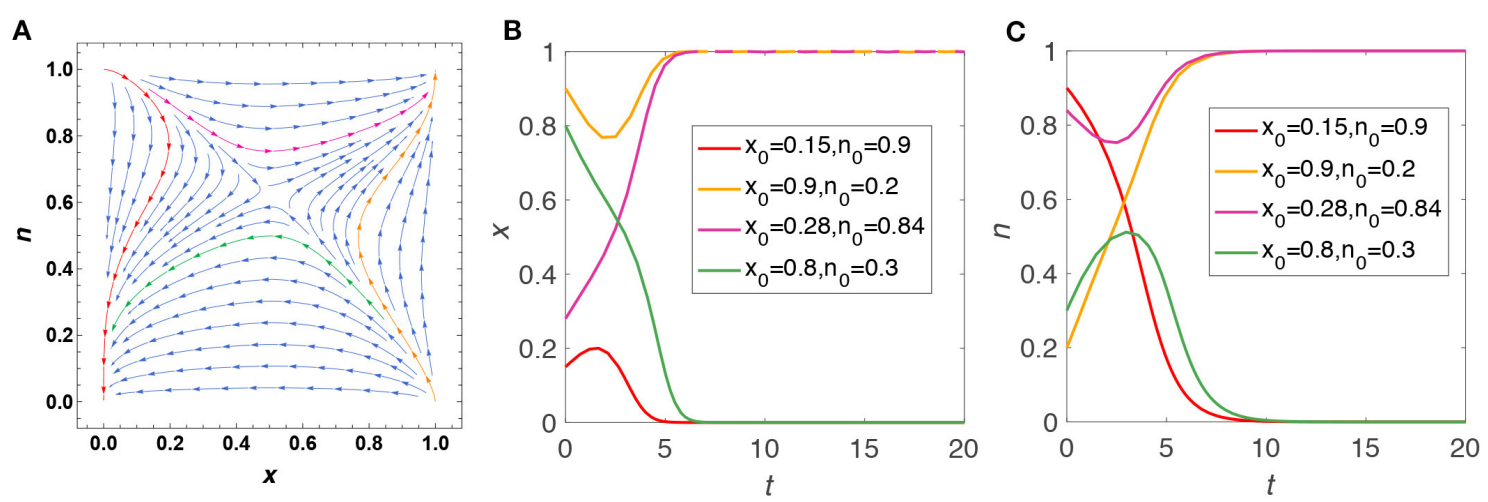

FIGURE 3 | A typical case in which the cooperation and the environment co-evolve toward either $(1,1)$ or $(0,0)$, indicating that the social innovation of new cognition succeeds or fails, respectively. We provide phase plane dynamics in (A) and highlight four typical trajectories in different colors. The detailed time evolutions of strategies and the environment for each trajectory are presented in (B,C), correspondingly. Parameters: $k_{1}=2, k_{2}=1, \theta=1, \epsilon=1, P_{1}=-0.5$.
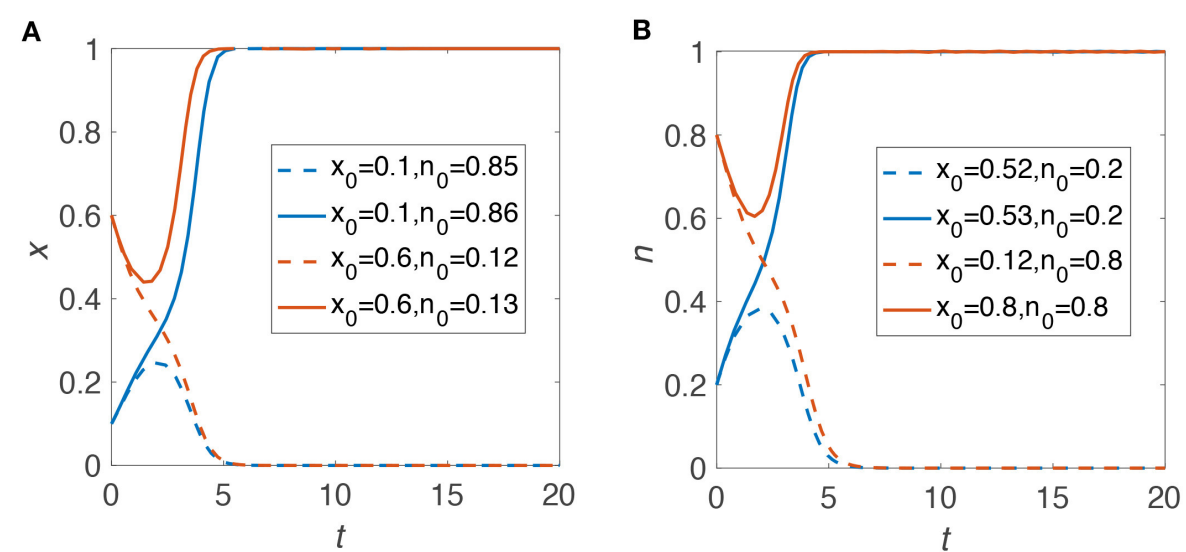

FIGURE 4 | Emergence of bifurcation phenomena: sensitivity to initial conditions. The existence of interior saddle points in $x$ - $n$ system can lead to "randomness" in real-world practice: either (A) a small disturbance in initial public opinion environment given a certain level of initial cooperation, or (B) a small disturbance in initial fraction of cooperators given a certain initial environment could change the evolutionary directions of the dynamical system. Parameters: $k_{1}=2, k_{2}=1, \theta=2, \epsilon=2$, $P_{1}=0.5$.

$$
\left\{\begin{array}{l}
x^{*}=\frac{1}{1+\theta} \\
n^{*}=\frac{k_{2}+\frac{\theta k_{1}}{1+\theta}-P_{1}}{k_{1}+2 k_{2}}
\end{array}\right.
$$

Within this interval, the boundary fixed point becomes an unstable equilibrium when $-\frac{k_{1}}{1+\theta}-k_{2}<P_{1}<-k_{2}$ and $k_{2}<$ $P_{1}<\frac{\theta k_{1}}{1+\theta}+k_{2}$, and vanishes when $-k_{2}<P_{1}<k_{2}$.

To summarize, we show that when the inherent payoff of adopting a certain cognition is large enough to ignore the environmental influence through public discourse, that cognition will eventually dominant the population with a fully supportive environment. Nevertheless, if the impact of public opinion environment is comparable to the inherent benefit from the competitive cognitions, there are opportunities for both cognitions to win, depending on initial conditions and model parameters.

\subsection{Evolutionary Dynamics With Interior Saddle Points}

We are particularly interested in exploring dynamical patterns under the existence of interior saddle points. In Figure 3, we show the phase plane dynamics of a typical case where the cooperation and the environment co-evolve toward either $(1,1)$ or $(0,0)$. Here, $k_{1}=2, k_{2}=1, \theta=1, \epsilon=1, P_{1}=-0.5$ and the interior fixed point is $\left(\frac{1}{2}, \frac{5}{8}\right)$ according to equation 6 . Four typical trajectories are highlighted in Figure $\mathbf{3 A}$ and the corresponding variations of strategies as well as the public opinion environment over time are provided in Figures 3B,C. We find that all trajectories experience two stages of dynamics. During the first stage, the cooperation and the environment evolve to opposite directions: either the supporters of new cognition increase 

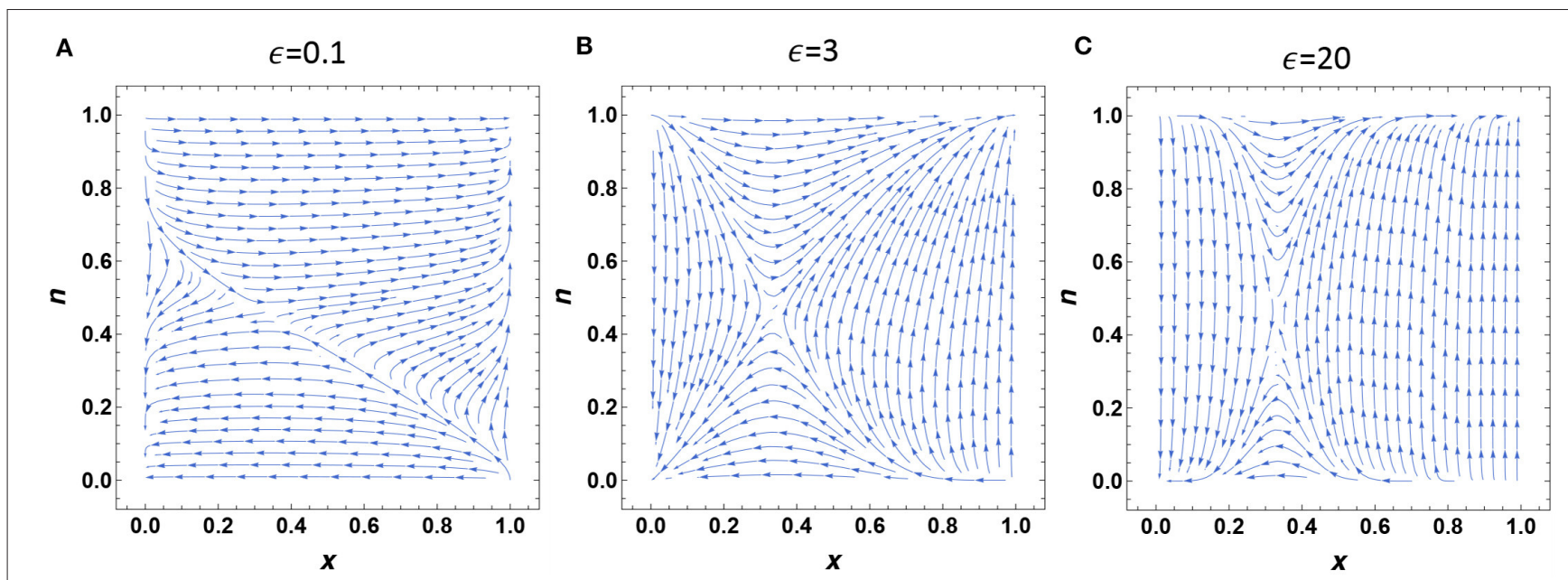

FIGURE 5 | Effects of the relative feedback speed. We fix $k_{1}=2, k_{2}=1, \theta=2, P_{1}=0.5$, and change $\epsilon=0.1,3,20$ in $(\mathbf{A}-\mathbf{C})$, respectively.

while the external environment becomes worse (pink and red trajectories), or the public opinion environment gets better while the supporters of new cognition become fewer (orange and green trajectories). During the second stage, on the other hand, the cooperation and the environment benefit from the mutual positive feedbacks and co-evolve toward the same direction: the population adopts the new cognition with a fully supportive environment (pink and orange trajectories) or adopts the old cognition with a fully unsupportive environment (red and green trajectories).

To conclude, the intuitive understanding of this dynamical character can be explained as follows. The eventual evolutionary direction of this system actually depends on the results of two types of "competition" at initial stage: (i) whether the increasing public opinion environment could save the loss of new cognition adopters (orange trajectory succeeds while green trajectory fails), and (ii) whether the increasing new cognition supporters would arouse enough external supports from media environment (pink trajectory succeeds while red trajectory fails).

Unfortunately, the existence of interior saddle point in co-evolution system leads to the emergence of bifurcation phenomena, as shown in Figure 4, which indicates that it is difficult to steer the social evolution to a desired direction. The parameters are $k_{1}=2, k_{2}=1, \theta=2, \epsilon=$ 2, $P_{1}=0.5$, and the $x-n$ phase diagram can be found in Figure 1. The final evolutionary direction, to some extent, can be significantly affected by "randomness" or "luckiness" in real-world practice: both a tiny disturbance in public opinion environment given a certain fraction of cooperators (Figure 4A) and a tiny disturbance in the fraction of cooperators given a certain level of environment (Figure 4B) could change the evolutionary directions of the dynamical system.

These dynamical properties just highlight the importance of the interior saddle point. We further analyze how our model parameters affect the position of the interior saddle point $\left(x^{*}, n^{*}\right)$, which determines the attraction basin of the two stable equilibriums $[(0,0)$ and $(1,1)]$. Generally, a larger $x^{*}$ or $n^{*}$ indicates that more initial cooperators or a more supportive initial environment is needed for successfully promoting the new cognition. According to equation $6, x^{*}$ is only influenced by $\theta$, the relative strength of enhancing the environment by cooperators vs. decreasing the environment by defectors. Obviously, $x^{*}$ increases as $\theta$ decreases, calling for more cooperators participating the social innovation if each of them has less impact. On the other hand, $n^{*}$ is influenced by four parameters: $\theta, P_{1}, k_{1}$ and $k_{2}$. It is easy to see that $n^{*}$ increases as $\theta$ increases or $P_{1}$ decreases, whereas $k_{1}$ and $k_{2}$ have more complicated influence. The formula of $n^{*}$ can be rewritten as

$$
n^{*}=\frac{\theta}{1+\theta}+\frac{\frac{1-\theta}{1+\theta} k_{2}-P_{1}}{k_{1}+2 k_{2}}
$$

Therefore, (i) when $P_{1}<\frac{1-\theta}{1+\theta} k_{2}, n^{*}$ increases as $k_{1}$ decreases; (ii) when $P_{1}=\frac{1-\theta}{1+\theta} k_{2}, n^{*}=\frac{\theta}{1+\theta}$; (iii) when $P_{1}>\frac{1-\theta}{1+\theta} k_{2}, n^{*}$ increases as $k_{1}$ increases. Similarly, we have

$$
n^{*}=\frac{1}{2}+\frac{\frac{\theta-1}{2(1+\theta)} k_{1}-P_{1}}{k_{1}+2 k_{2}}
$$

Hence, (i) when $P_{1}<\frac{\theta-1}{2(1+\theta)} k_{1}, n^{*}$ increases as $k_{2}$ decreases; (ii) when $P_{1}=\frac{\theta-1}{2(1+\theta)} k_{1}, n^{*}=\frac{1}{2}$; (iii) when $P_{1}>\frac{\theta-1}{2(1+\theta)} k_{1}, n^{*}$ increases as $k_{2}$ increases.

Furthermore, we show the impact of the relative feedback speed in Figure 5. We fix $k_{1}=2, k_{2}=1, \theta=2, P_{1}=0.5$, and change $\epsilon=0.1,3,20$ from Figures 5A-C. Aside from changing the convergence speed of the dynamical system, we find the relative feedback speed can also influence the attraction basin of the two stable states, though not affecting the position of interior saddle point. A larger $\epsilon$ gives advantage to the environmental feedback compared to the strategy dynamics, resulting in the occurrence of more steep trajectories. 


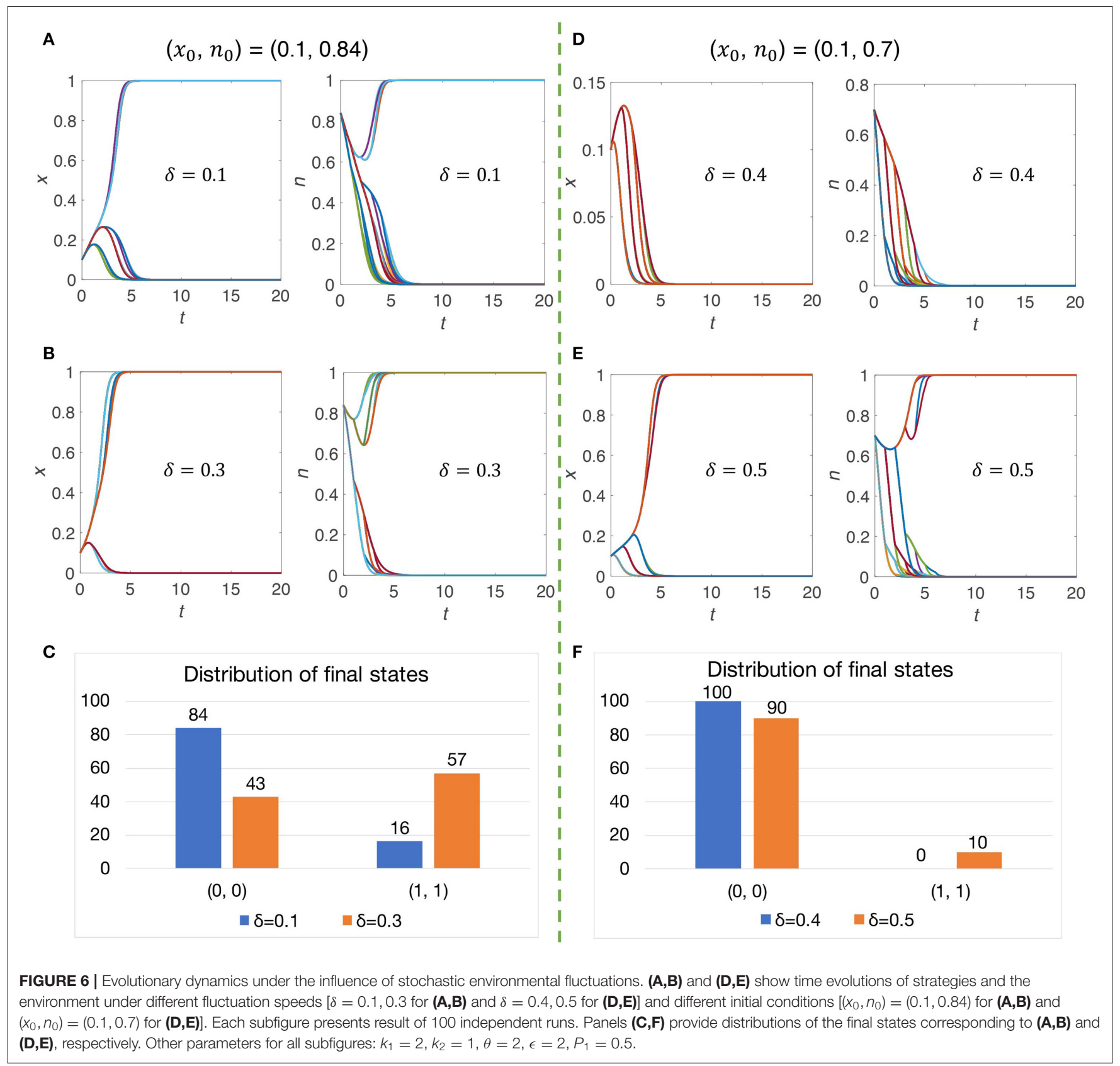

\subsection{Impact of Stochastic Environmental Fluctuations}

While the power of external stochasticity (via direct disturbance on either strategy or environment) has been stressed in Figure 4, it is meaningful to further explore the influence of systematic stochasticity on our deterministic model. Here, we consider a simple stochastic fluctuation embedded in environmental evolution, which is given by

$$
\dot{n}=\epsilon n(1-n)\left((\theta x-(1-x))+\phi_{T}(\delta)\right)
$$

where $\phi_{T}(\delta)=\delta$ or $-\delta$ with the same probability 0.5 at each time interval $[T, T+1), T \in\{0,1,2, \ldots\}$, and $\delta>0$ describes the relative fluctuation speed of the environment.

In Figure 6, we study the impact of stochastic environmental fluctuations in two scenarios: (i) the initial state $\left(x_{0}, n_{0}\right)=$ $(0.1,0.84)$ is close to the boundary of the two attraction basins (Figures 6A-C), or (ii) the initial state $\left(x_{0}, n_{0}\right)=(0.1,0.7)$ locates inside the attraction basin of $(0,0)$ and is relatively far away from the boundary (Figures 6D-F). Note that the actual changing speed of the environment caused by the systematic stochasticity at time $t$ can be calculated by $\epsilon n_{t}\left(1-n_{t}\right) \phi_{t}(\delta)$, which is small under all our parameters. In scenario (i), we find that even a tiny stochastic fluctuation is capable of changing the co-evolutionary direction, i.e., driving the $x-n$ system toward $(1,1)$ instead 
of $(0,0)$. In addition, a larger $\delta$ significantly promotes such changes, bring about more $(1,1)$ states when system becomes stable. In scenario (ii), however, the final evolutionary results can be changed only when $\delta$ is large enough.

\section{CONCLUSIONS AND DISCUSSIONS}

Evolutionary game theory is an effective approach to study the incentive-driven dynamical evolutions, where different strategies "compete" for a higher payoff in order to survive in the natural systems $[48,49]$. In particular, recent advance highlights the importance of coupled interactions between strategies and external environment [50]. The strategydependent environmental feedbacks give rise to the oscillating dynamics of both cooperation and environment states, which provides profound insights toward many important issues, such as overexploitation problem [51], social distancing behavior during COVID-19 pandemic [31], vaccine refusal [52-54], etc. Further studies show that such co-evolution could lead to the emergence of persistent cooperation if the relative feedback speed exceeds a threshold, breaking the tragedy of the commons in traditional public goods game [55]. Despite the progress, few works have extended this powerful theoretical framework to the field of cognition (information, beliefs, ideas, behaviors, etc.) evolution. Specifically, it remains largely unknown how public opinion environment influences the competition of two opposite cognitions, which is ubiquitous and is of vital importance in a polarizing society [56].

In this paper, we introduce this co-evolutionary game theory into our new model to study the dynamics of feedback loops between competitive cognitions and public opinion environment. We find that when the inherent payoff or loss from the new cognition exceeds a critical value with regard to the environmental impact, individuals will neglect the feedbacks from public opinion and the population will be dominated by one cognition regardless of initial conditions. Otherwise, a saddle point occurs in the system and both cooperation (the new cognition) domination with a fully supportive environment and defection (the old cognition) domination with a fully unsupportive environment are stable states. Under this circumstance, the final result of the competition depends on the parameters and initial conditions. A further exploration on dynamical patterns under the existence of interior saddle point is also performed. Results unveil the sensitivity of the

\section{REFERENCES}

1. Vespignani A. Modelling dynamical processes in complex socio-technical systems. Nat Phys. (2012) 8:32-9. doi: 10.1038/ nphys 2160

2. Axelrod R. The dissemination of culture: a model with local convergence and global polarization. J Confl Resol. (1997) 41:203-26. doi: 10.1177/0022002797041002001

3. Deffuant G, Neau D, Amblard F, Weisbuch G. Mixing beliefs among interacting agents. Adv Complex Syst. (2000) 3:87-98. doi: 10.1142/S0219525900000078 system to tiny disturbance in some areas, indicating that the eventual evolutionary direction of the competitive cognition sometimes might be determined by "randomness" or "luckiness" in real world. Similar phenomena are also observed when a simple systematic stochastic fluctuation is considered in the environmental evolution. Finally, we show that the relative feedback speed not only influences the convergence speed, but also changes the attraction basin of the two stable states.

A noteworthy limitation of our work is that our model cannot reproduce the possible stable state where the two cognitions both survive. Previous works have revealed many mechanisms that contribute to the coexistence in competitive dynamics or the emergence of persistent cooperation, such as the existence of inactive individuals [57], the network structures [58-62], the homogeneous trends [26], etc. [6368]. Further studies may consider the incorporation of these influential factors based on our heuristic modeling framework.

\section{DATA AVAILABILITY STATEMENT}

The original contributions presented in the study are included in the article/Supplementary Material, further inquiries can be directed to the corresponding author/s.

\section{AUTHOR CONTRIBUTIONS}

HL conceived the project. XW conceived the model. HL, XW, and LL performed the analysis and wrote the paper. ZL provided funding and supervised the research. All authors contributed to the article and approved the submitted version.

\section{FUNDING}

This work was supported in part by the National Natural Science Foundation of China (Grant Nos. U1636211, 61672081, 61370126), and the Fund of the State Key Laboratory of Software Development Environment (Grant No. SKLSDE-2019ZX-17).

\section{SUPPLEMENTARY MATERIAL}

The Supplementary Material for this article can be found online at: https://www.frontiersin.org/articles/10.3389/fphy. 2021.658130/full\#supplementary-material 
8. Festinger L. Cognitive dissonance. Sci Am. (1962) 207:93-106. doi: 10.1038/scientificamerican1062-93

9. Del Vicario M, Bessi A, Zollo F, Petroni F, Scala A, Caldarelli G, et al. The spreading of misinformation online. Proc Natl Acad Sci USA. (2016) 113:554-9. doi: 10.1073/pnas.1517441113

10. Sasahara K, ChenW, Peng H, Ciampaglia GL, Flammini A, Menczer F. Social influence and unfollowing accelerate the emergence of echo chambers. $J$ Comput Soc Sci. (2020) 4:381-402. doi: 10.1007/s42001-020-00084-7

11. Cota W, Ferreira SC, Pastor-Satorras R, Starnini M. Quantifying echo chamber effects in information spreading over political communication networks. EPJ Data Sci. (2019) 8:35. doi: 10.1140/epjds/s13688-019-0213-9

12. Baumann F, Lorenz-Spreen P, Sokolov IM, Starnini M. Modeling echo chambers and polarization dynamics in social networks. Phys Rev Lett. (2020) 124:048301. doi: 10.1103/PhysRevLett.124.048301

13. Choi D, Chun S, Oh H, Han J, Kwon TT. Rumor propagation is amplified by echo chambers in social media. Sci Rep. (2020) 10:1-10. doi: 10.1038/s41598-019-57272-3

14. Wang X, Sirianni AD, Tang S, Zheng Z, Fu F. Public discourse and social network echo chambers driven by socio-cognitive biases. Phys Rev X. (2020) 10:041042. doi: 10.1103/PhysRevX.10.041042

15. Wang Z, Wang L, Szolnoki A, Perc M. Evolutionary games on multilayer networks: a colloquium. Eur Phys J B. (2015) 88:1-15. doi: 10.1140/epjb/e2015-60270-7

16. Boccaletti S, Bianconi G, Criado R, Del Genio CI, Gómez-Gardenes J, Romance M, et al. The structure and dynamics of multilayer networks. Phys Rep. (2014) 544:1-122. doi: 10.1016/j.physrep.2014.07.001

17. Wang X, Li W, Liu L, Pei S, Tang S, Zheng Z. Promoting information diffusion through interlayer recovery processes in multiplex networks. Phys Rev E. (2017) 96:032304. doi: 10.1103/PhysRevE.96.032304

18. Chen W, Yang Z, Wu T. Evolution of cooperation driven by collective interdependence on multilayer networks. Appl Math Comput. (2021) 388:125532. doi: 10.1016/j.amc.2020.125532

19. Liu L, Wang X, Tang S, Zheng Z. Complex social contagion induces bistability on multiplex networks. arXiv preprint arXiv:2005.00664 (2020).

20. Granell C, Gómez S, Arenas A. Dynamical interplay between awareness and epidemic spreading in multiplex networks. Phys Rev Lett. (2013) 111:128701. doi: 10.1103/PhysRevLett.111.128701

21. De Domenico M, Solé-Ribalta A, Cozzo E, Kivelä M, Moreno Y, Porter MA, et al. Mathematical formulation of multilayer networks. Phys Rev X. (2013) 3:041022. doi: 10.1103/PhysRevX.3.041022

22. Gomez S, Diaz-Guilera A, Gomez-Gardenes J, Perez-Vicente CJ, Moreno Y, Arenas A. Diffusion dynamics on multiplex networks. Phys Rev Lett. (2013) 110:028701. doi: 10.1103/PhysRevLett.110.028701

23. $\mathrm{Hu} \mathrm{Y}$, Havlin S, Makse HA. Conditions for viral influence spreading through multiplex correlated social networks. Phys Rev X. (2014) 4:021031. doi: 10.1103/PhysRevX.4.021031

24. Gleeson JP, Ward JA, O'sullivan KP, Lee WT. Competition-induced criticality in a model of meme popularity. Phys Rev Lett. (2014) 112:048701. doi: 10.1103/PhysRevLett.112.048701

25. Chang HCH, Fu F. Co-diffusion of social contagions. N J Phys. (2018) 20:095001. doi: 10.1088/1367-2630/aadce7

26. Liu L, Wang X, Zheng Y, Fang W, Tang S, Zheng Z. Homogeneity trend on social networks changes evolutionary advantage in competitive information diffusion. N J Phys. (2020) 22:013019. doi: 10.1088/1367-2630/ $\mathrm{ab} 623 \mathrm{c}$

27. Grinberg N, Joseph K, Friedland L, Swire-Thompson B, Lazer D. Fake news on twitter during the 2016 us presidential election. Science. (2019) 363:374-8. doi: $10.1126 /$ science.aau2706

28. Aral S, Eckles D. Protecting elections from social media manipulation. Science. (2019) 365:858-61. doi: 10.1126/science.aaw8243

29. Weitz JS, Park SW, Eksin C, Dushoff J. Awareness-driven behavior changes can shift the shape of epidemics away from peaks and toward plateaus, shoulders, and oscillations. Proc Natl Acad Sci USA. (2020) 117:32764-71. doi: 10.1073/pnas.2009911117

30. Johnson NF, Velásquez N, Restrepo NJ, Leahy R, Gabriel N, El Oud S, et al. The online competition between pro-and anti-vaccination views. Nature. (2020) 582:1-4. doi: 10.1038/s41586-020-2281-1
31. Glaubitz A, Fu F. Oscillatory dynamics in the dilemma of social distancing. Proc R Soc A. (2020) 476:20200686. doi: 10.1098/rspa.2020.0686

32. Vosoughi S, Roy D, Aral S. The spread of true and false news online. Science. (2018) 359:1146-51. doi: 10.1126/science.aap9559

33. Karrer B, Newman ME. Competing epidemics on complex networks. Phys Rev E. (2011) 84:036106. doi: 10.1103/PhysRevE.84.0 36106

34. Sanz J, Xia CY, Meloni S, Moreno Y. Dynamics of interacting diseases. Phys Rev X. (2014) 4:041005. doi: 10.1103/PhysRevX.4.0 41005

35. Leventhal GE, Hill AL, Nowak MA, Bonhoeffer S. Evolution and emergence of infectious diseases in theoretical and real-world networks. Nat Commun. (2015) 6:6101. doi: 10.1038/ncomms7101

36. Stanoev A, Trpevski D, Kocarev L. Modeling the spread of multiple concurrent contagions on networks. PLoS ONE. (2014) 9:e95669. doi: 10.1371/journal.pone.0095669

37. Jie R, Qiao J, Xu G, Meng Y. A study on the interaction between two rumors in homogeneous complex networks under symmetric conditions. Phys A Stat Mech Appl. (2016) 454:129-42. doi: 10.1016/j.physa.2016.02.048

38. Centola D, Macy M. Complex contagions and the weakness of long ties. Am J Sociol. (2007) 113:702-34. doi: 10.1086/521848

39. Stewart AJ, Mosleh M, Diakonova M, Arechar AA, Rand DG, Plotkin JB. Information gerrymandering and undemocratic decisions. Nature. (2019) 573:117-21. doi: 10.1038/s41586-019-1507-6

40. Johnson N, Leahy R, Restrepo NJ, Velasquez N, Zheng M, Manrique P, et al. Hidden resilience and adaptive dynamics of the global online hate ecology. Nature. (2019) 573:261-5. doi: 10.1038/s41586-019-1494-7

41. Gore J, Youk H, Van Oudenaarden A. Snowdrift game dynamics and facultative cheating in yeast. Nature. (2009) 459:253-6. doi: 10.1038/nature07921

42. Sanchez A, Gore J. Feedback between population and evolutionary dynamics determines the fate of social microbial populations. PLoS Biol. (2013) 11:e1001547. doi: 10.1371/journal.pbio.1001547

43. Perc M, Szolnoki A. Coevolutionary games-a mini review. Biosystems. (2010) 99:109-25. doi: 10.1016/j.biosystems.2009.10.003

44. Weitz JS, Eksin C, Paarporn K, Brown SP, Ratcliff WC. An oscillating tragedy of the commons in replicator dynamics with game-environment feedback. Proc Natl Acad Sci USA. (2016) 113:E7518-25. doi: 10.1073/pnas.1604096113

45. Tilman AR, Plotkin JB, Akçay E. Evolutionary games with environmental feedbacks. Nat Commun. (2020) 11:1-11. doi: 10.1038/s41467-020-14531-6

46. Wang $\mathrm{X}$, Zheng $\mathrm{Z}$, Fu F. Steering eco-evolutionary game dynamics with manifold control. Proc $R$ Soc A. (2020) 476:20190643. doi: 10.1098/rspa.2019.0643

47. Hilbe $\mathrm{C}, \mathrm{Wu} \mathrm{B}$, Traulsen $\mathrm{A}$, Nowak MA. Cooperation and control in multiplayer social dilemmas. Proc Natl Acad Sci USA. (2014) 111:16425-30. doi: 10.1073/pnas.1407887111

48. Nowak MA. Evolutionary Dynamics: Exploring the Equations of Life Cambridge, MA: Harvard University Press (2006). doi: 10.2307/j.ctvjghw98

49. West SA, Griffin AS, Gardner A, Diggle SP. Social evolution theory for microorganisms. Nat Rev Microbiol. (2006) 4:597-607. doi: $10.1038 /$ nrmicrol461

50. Wang $\mathrm{X}, \mathrm{Fu}$ F. Eco-evolutionary dynamics with environmental feedback: cooperation in a changing world. EPL. (2020) 132:10001. doi: 10.1209/0295-5075/132/10001

51. Chen X, Szolnoki A. Punishment and inspection for governing the commons in a feedback-evolving game. PLoS Comput Biol. (2018) 14:e1006347. doi: 10.1371/journal.pcbi.1006347

52. $\mathrm{Wu} \mathrm{B}, \mathrm{Fu} \mathrm{F}$, Wang $\mathrm{L}$. Imperfect vaccine aggravates the long-standing dilemma of voluntary vaccination. PLoS ONE. (2011) 6:e20577. doi: 10.1371/journal.pone.0020577

53. Wei $\mathrm{Y}$, Lin $\mathrm{Y}, \mathrm{Wu}$ B. Vaccination dilemma on an evolving social network. $J$ Theoret Biol. (2019) 483:109978. doi: 10.1016/j.jtbi.2019.08.009

54. Chen X, Fu F. Imperfect vaccine and hysteresis. Proc R Soc B Biol Sci. (2019) 286:20182406. doi: 10.1098/rspb.2018.2406

55. Shao Y, Wang X, Fu F. Evolutionary dynamics of group cooperation with asymmetrical environmental feedback. EPL. (2019) 126:40005. doi: 10.1209/0295-5075/126/40005 
56. Macy M, Deri S, Ruch A, Tong N. Opinion cascades and the unpredictability of partisan polarization. Sci Adv. (2019) 5:eaax0754. doi: 10.1126/sciadv.aax0754

57. Hauert C, Holmes M, Doebeli M. Evolutionary games and population dynamics: maintenance of cooperation in public goods games. Proc $R$ Soc $B$ Biol Sci. (2006) 273:2565-71. doi: 10.1098/rspb.2006.3600

58. Ohtsuki H, Nowak MA. Evolutionary stability on graphs. J Theoret Biol. (2008) 251:698-707. doi: 10.1016/j.jtbi.2008.01.005

59. Tarnita CE, Antal T, Ohtsuki H, Nowak MA. Evolutionary dynamics in set structured populations. Proc Natl Acad Sci USA. (2009) 106:8601-4. doi: 10.1073/pnas.0903019106

60. Ohtsuki H, Nowak MA, Pacheco JM. Breaking the symmetry between interaction and replacement in evolutionary dynamics on graphs. Phys Rev Lett. (2007) 98:108106. doi: 10.1103/PhysRevLett.98. 108106

61. Wang Z, Xia C, Chen Z, Chen G. Epidemic propagation with positive and negative preventive information in multiplex networks. IEEE Trans Cybernet. (2021) 51:1454-62. doi: 10.1109/TCYB.2019. 2960605

62. Jian Q, Li X, Wang J, Xia C. Impact of reputation assortment on tagmediated altruistic behaviors in the spatial lattice. Appl Math Comput. (2021) 396:125928. doi: 10.1016/j.amc.2020.125928

63. Fu F, Hauert C, Nowak MA, Wang L. Reputation-based partner choice promotes cooperation in social networks. Phys Rev E. (2008) 78:026117. doi: 10.1103/PhysRevE.78.026117
64. Fu F, Wu T, Wang L. Partner switching stabilizes cooperation in coevolutionary prisoner's dilemma. Phys Rev E. (2009) 79:036101. doi: 10.1103/PhysRevE.79.036101

65. Chen X, Szolnoki A, Perc M. Competition and cooperation among different punishing strategies in the spatial public goods game. Phys Rev E. (2015) 92:012819. doi: 10.1103/PhysRevE.92.012819

66. Szolnoki A, Chen X. Gradual learning supports cooperation in spatial prisoner's dilemma game. Chaos Solitons Fractals. (2020) 130:109447. doi: 10.1016/j.chaos.2019.109447

67. Wu T, Fu F, Dou P, Wang L. Social influence promotes cooperation in the public goods game. Phys A Stat Mech Appl. (2014) 413:86-93. doi: 10.1016/j.physa.2014.06.040

68. Hilbe C, Šimsa Š, Chatterjee K, Nowak MA. Evolution of cooperation in stochastic games. Nature. (2018) 559:246-9. doi: 10.1038/s41586-018-0277-x

Conflict of Interest: The authors declare that the research was conducted in the absence of any commercial or financial relationships that could be construed as a potential conflict of interest.

Copyright (C) 2021 Liu, Wang, Liu and Li. This is an open-access article distributed under the terms of the Creative Commons Attribution License (CC BY). The use, distribution or reproduction in other forums is permitted, provided the original author(s) and the copyright owner(s) are credited and that the original publication in this journal is cited, in accordance with accepted academic practice. No use, distribution or reproduction is permitted which does not comply with these terms. 